\title{
Long-term treatment of bipolar disorder with a radioelectric asymmetric conveyor
}

\author{
This article was published in the following Dove Press journal: \\ Neuropsychiatric Disease and Treatment \\ 14 June 201 I \\ Number of times this article has been viewed
}

Piero Mannu'

Salvatore Rinaldi ${ }^{1,2}$

Vania Fontani'

Alessandro Castagna'

'Rinaldi-Fontani Institute,

Department of Neuro-Psycho-Physio Pathology, Florence, Italy; ${ }^{2}$ School of Occupational Medicine, University of Florence, Florence, Italy
Correspondence: Salvatore Rinaldi Rinaldi-Fontani Institute, Viale Belfiore 43, 50144 Florence, Italy

Tel +390 55290307

Fax +390 55290399

Email srinaldi@irf.it
Background: The bipolar spectrum disorders are considered an important and frequent psychiatric problem. The clinical complexity of these illnesses due to the coexistence of depressive and excitative phases is correlated with the global difficulty of adequate treatment; consequently, the prognosis is not optimal. For this reason, in recent years, novel nonpharmacologic physical approaches have been tested for bipolar disorders, with encouraging results. The aim of this study was to evaluate the long-term effectiveness of a radioelectric asymmetric brain stimulation device associated with lithium (REAC-lithium) versus previous treatments in subjects with bipolar disorder I or II, evaluated as the number of recurrences compared with the period of illness preceding treatment with REAC-lithium.

Methods: The charts of 56 bipolar patients attending our institute were retrospectively evaluated. Treatment with REAC-lithium was administered following the standard Rinaldi-Fontani Institute protocol. Add-on treatments were allowed in the event of manic or depressive recurrence. Eight patients (Group 1) were followed for $30.2 \pm 3.0$ months, 14 patients (Group 2) were followed for $25.3 \pm 3.3$ months, 25 patients (Group 3) were followed for $20.3 \pm 1.6$ months, and nine patients (Group 4) were followed for $16.2 \pm 0.5$ months.

Results: After REAC-lithium treatment, the number of manic and depressive episodes in Group 1 decreased from $2.1 \pm 0.6$ and $3.0 \pm 0.7$ to $0.12 \pm 0.0$ and $0.8 \pm 0.4$, respectively. In Group 2, the number of manic and depressive episodes decreased from $2.4 \pm 0.6$ and $3.9 \pm 0.7$ to $0.14 \pm 0.2$ and $0.0 \pm 0.0$, respectively. In Group 3, the number of manic and depressive episodes decreased from $2.6 \pm 0.8$ and $3.6 \pm 0.9$ to $0.04 \pm 0.0$ and $0.0 \pm 0.0$, respectively. In Group 4, the number of manic and depressive episodes decreased from $2.6 \pm 1.1$ and $3.7 \pm 1.0$ to $0.1 \pm 0.0$ and $0.0 \pm 0.0$, respectively. All results were statistically significant.

Conclusion: REAC showed good efficacy in treating both the manic and depressive phases of bipolar disorder, and in the prevention of recurrences/relapses.

Keywords: bipolar disorder, stress, radioelectric asymmetric brain stimulation

\section{Introduction}

The concept of the "bipolar spectrum" as a consequence of more accurate diagnostic assessment has progressively increased the classic epidemiologic boundary of bipolar disorder, ${ }^{1}$ historically affecting $0.8 \%-1.5 \%{ }^{2}$ to $15 \%{ }^{3}$ of the general population. In the same way, the complexity of the illness has posed the need for dimensional (cross-sectional and longitudinal) monitoring of the patient, in order to design more personalized and phase-specific therapeutic strategies. Thus, from a therapeutic point of view, the classic drugs for bipolar disorder (lithium, carbamazepine, valproic acid, antidepressants, and neuroleptics $)^{4}$ have been progressively supported with the new 
generation of anticonvulsants (topiramate, gabapentin, pregabalin, lamotrigine, and levetiracetam), ${ }^{5,6}$ and the atypical antipsychotics. ${ }^{7,8}$ Although the excitative phases (manic and hypomanic) are usually considered as the organizing principle of bipolar disorder, ${ }^{4}$ pooled data for the disease course show that, with exclusion of free intervals, nearly $70 \%$ of the "bipolar life" consists of depressive episodes and the remaining $30 \%$ consists of excitative phenomena. ${ }^{9,10}$ The main result of this hierarchical approach, based on the course of bipolar disorder, is a different pharmacologic treatment strategy for the manic phenomena and for bipolar depression. ${ }^{11,12}$ Despite recent efforts, ${ }^{13}$ the results of treatment of bipolar depression are not encouraging. ${ }^{14}$ The use of lamotrigine, after an initial enthusiastic introduction, has gradually reduced as an acute antidepressant in patients with bipolar disorder, and is currently used for the prevention of depressive recurrences. ${ }^{15-17}$ New pharmacologic data for atypical antipsychotics could be considered promising. These drugs seem to be useful in the depressive phase (without a manic switch) and in the manic phase, avoiding the depressive and apathetic motivational risk associated with the administration of typical antipsychotics. Unfortunately, the data available regarding the role of these drugs in the maintenance of bipolar disorder are fewer than expected. ${ }^{8}$ Moreover, it is important to emphasize that lithium remains the first-choice drug for bipolar disorder. However, because of the demonstrated and specific antimanic effect, the attempt to avoid the excited phases of bipolar disorder often produces a stabilization of patient mood, with a progressive deterioration of quality of life and reduced energy levels and motivation. ${ }^{18,19}$ Beyond psychopharmacologic treatment, other therapeutic strategies for bipolar disorder are available. The most studied of these is electroconvulsant therapy, probably the most effective treatment in the overall management of bipolar disorder, and in recent years, growing data favor transcranic magnetic stimulation. The radioelectric asymmetric conveyor (REAC) is a radiofrequency medical device based on an innovative technology for brain stimulation. REAC treatment is a novel approach for treatment of several mental disorders, and the results over time are very encouraging. ${ }^{20-25}$ The aim of this study was to evaluate the long-term effectiveness of REAC treatment associated with lithium (REAC-lithium) versus previous treatments in subjects with bipolar disorder I and II, evaluated as the number of recurrences compared with the period of illness preceding REAC-lithium treatment.

\section{Methods and materials}

The charts of 56 patients attending the Rinaldi-Fontani Institute in Florence and the Centro di Studi Psichici in
Cagliari were retrospectively evaluated. Patients diagnosed with bipolar disorder I or II according to the revised fourth edition of the Diagnostic Statistical Manual for Mental Disorders (DSM-IVTR) criteria were included. Inclusion criteria were as follows: both genders; age 18-65 years; written informed consent obtained; history of at least 12 months of psychopharmacologically treated bipolar disorder I/II; and at least four cycles of REAC treatment. Exclusion criteria included a history of seizures, head trauma, or brain disease, and evidence of chronic or severe disease (renal or hepatic impairment, or cancer). REAC treatment was administered following the standard Rinaldi-Fontani Institute schedule of one session of neuropostural optimization followed by 18 sessions of neuropsychophysical optimization. Add-on treatments were allowed in the event of manic or depressive recurrence. Patients were divided into four follow-up groups before and after REAC-lithium treatment, as follows: at least 72 months (Group 1); at least 48 months (Group 2); at least 36 months (Group 3); and at least 18 months (Group 4). Information on demographic variables, psychiatric history, concomitant treatments, and comorbid disorders were collected for each patient. The evaluation of REAC-lithium effectiveness was made by collecting the recurrence of mood episodes (manic or depressive according to DSM IV-TR). The Structured Clinical Interview for Diagnosis, the 21-item Hamilton Depression Rating Scale, and the Young Mania Rating Scale (YMRS) were been used. Statistical analysis was performed using a $t$-test for comparative groups, and all results with a $P<0.05$ were considered significant. Tolerability and safety were evaluated by collecting the reports of adverse events. Demographic variables and the psychiatric-psychopharmacologic history of the patients are reported in Table 1. Eight patients (Group 1) were followed for $30.2 \pm 3.0$ months, 14 patients (Group 2) for $25.3 \pm 3.3$ months, 25 patients (Group 3 ) for $20.3 \pm 1.6$ months, and nine patients (Group 4) for $16.2 \pm 0.5$ months. Lithium was dosed at $980.0 \pm 135.6 \mathrm{mg} /$ day, $825.0 \pm 50.0 \mathrm{mg} /$ day, $850.5 \pm 100.0 \mathrm{mg} /$ day, and $950.5 \pm 75.5 \mathrm{mg} /$ day in the four groups of patients, respectively. The demographic variables and psychiatric history of the patients are reported in Table 1.

$\mathrm{REAC}^{26,27}$ is a medical device based on innovative technology for biostimulation. REAC works with a typical range frequency of $2.4,5.8$, or $10.5 \mathrm{gHz}$, as selected by the operator for each specific protocol used. A frequency of $10.5 \mathrm{gHz}$ was used in the brain stimulation protocols used in this study, with a specific absorption rate ${ }^{28-30}$ of $7 \mu \mathrm{W} / \mathrm{kg}$. REAC treatments have proven efficacy in ameliorating stress-related disorders, depression, and anxiety. ${ }^{20-25}$ The REAC pulse used in this 
Table I Overall demographic variables and psychiatric history before REAC-lithium treatment

\begin{tabular}{|c|c|}
\hline \multicolumn{2}{|l|}{ Gender, n (\%) } \\
\hline Male & $28(50.0)$ \\
\hline Female & $28(50.0)$ \\
\hline \multicolumn{2}{|l|}{ Age, years } \\
\hline Mean (SD) & $29.5(3.9)$ \\
\hline Range & $25-32$ \\
\hline \multicolumn{2}{|l|}{ Onset age, years } \\
\hline Mean (SD) & $22.3(3.6)$ \\
\hline Range & $19-26$ \\
\hline \multicolumn{2}{|l|}{ Diagnosis, n (\%) } \\
\hline BD I & $22(39.3)$ \\
\hline BD II & $34(60.7)$ \\
\hline \multicolumn{2}{|l|}{ Type of onset, $n$ (\%) } \\
\hline Manic & $20(35.7)$ \\
\hline Depressive & $36(64.3)$ \\
\hline \multicolumn{2}{|l|}{ Episodes/patient mean (SD) } \\
\hline Manic & $2.4(0.8)$ \\
\hline Depressive & $3.6(0.8)$ \\
\hline \multicolumn{2}{|l|}{ Hospitalization, $n$} \\
\hline Reason: Excitative episode & 6 \\
\hline Depressive episode & 19 \\
\hline \multicolumn{2}{|l|}{ Previous treatments, $\mathrm{n}$} \\
\hline Lithium alone & 26 \\
\hline Carbamazepine & 5 \\
\hline Olanzapine & I \\
\hline Lithium + valproate & 12 \\
\hline Lithium + olanzapine & 5 \\
\hline Lithium + SSRI & 5 \\
\hline Lithium + imipramine & 2 \\
\hline
\end{tabular}

Abbreviations: $\mathrm{BD}$, bipolar disorder; SD, standard deviation; SSRI, selective serotonin reuptake inhibitor.

study was a 7 radiofrequency burst of $500 \mathrm{msec}$, applied by touching the metallic tip of the REAC probe to the ear pavilion using neuropostural optimization and neuropsychophysical optimization protocols. The treatments are painless, noninvasive, and without negative adverse effects.

\section{Results}

\section{Before REAC-lithium therapy}

Mean baseline and onset ages for Group 1 (seven males and one female; all bipolar disorder I) were $31.4 \pm 2.9$ years and $23.9 \pm 2.7$ years, respectively. The mean numbers of previous manic and depressive episodes per patient were $2.1 \pm 0.6$ and $3.0 \pm 0.7$, respectively, and the overall number of hospitalizations was seven (five due to depressive episodes and two due to a manic mood state). Bipolar disorder onset was depressive in three patients and manic in five patients. All patients in Group 1 had comorbid pathologic gambling. Lithium was satisfactory for mood disorder, but ineffective for pathologic gambling. The switch to REAC-lithium treatment occurred during a depressive phase in three subjects and during the depression-free period in the remaining four patients. Mean baseline and onset ages for Group 2 (seven males [four bipolar disorder I and three bipolar disorder II], and seven females [all bipolar disorder II]) were $29.9 \pm 5.0$ years and $21.2 \pm 4.2$ years, respectively. Mean numbers of previous manic and depressive episodes per patient were $2.4 \pm 0.6$ and $3.9 \pm 0.7$, respectively, with an overall number of nine hospitalizations for depressive episodes. The onset of bipolar disorder was depressive in nine patients and manic in five patients. Patients in Group 2 were treated with lithium alone $(n=6)$, lithium added on to valproate $(n=3)$, lithium added on to olanzapine $(n=2)$, lithium added on to fluoxetine ( $\mathrm{n}=1)$, and carbamazepine $(n=2)$. The switch to REAC-lithium treatment occurred during a depressive phase in nine subjects and during the free period in the remaining five patients.

Mean baseline and onset ages for Group 3 (11 males [four bipolar disorder I and seven bipolar disorder II], and 14 females [three bipolar disorder I and 11 bipolar disorder II]) were $28.3 \pm 3.7$ years and $21.0 \pm 3.5$ years, respectively. The mean numbers of previous manic and depressive episodes per patient were $2.6 \pm 0.8$ and $3.6 \pm 0.9$, respectively, with an overall number of seven hospitalizations (four for depressive episodes and three for manic episodes). The onset of bipolar disorder was depressive in 17 patients and manic in eight patients. Ten patients were treated with lithium alone, six with lithium added on to valproate, three with lithium added on to olanzapine, one with lithium added on to sertraline, two with lithium added on to imipramine, and three with carbamazepine. The switch to REAC-lithium treatment occurred during a depressive phase in 15 subjects and during the free period in the remaining 10 patients.

Mean baseline and onset ages for Group 4 (three males [one bipolar disorder I and two bipolar disorder II], and six females [two bipolar disorder I and four bipolar disorder II]) were $30.0 \pm 3.9$ years and $23.1 \pm 4.0$ years, respectively. The mean number of previous manic and depressive episodes were $2.6 \pm 1.1$ and $3.7 \pm 1.0$, respectively, with an overall number of two hospitalizations (one due to a depressive episode and one due to a manic episode). The onset of bipolar disorder was depressive in seven patients and manic in two patients. Previous treatments were as follows: lithium alone $(\mathrm{n}=2)$; lithium added on to valproate $(\mathrm{n}=3)$; olanzapine $(\mathrm{n}=1)$; and lithium added on to citalopram $(\mathrm{n}=3)$. The switch to REAC-lithium treatment occurred during a depressive phase in five subjects and during the free period in the remaining four patients. 


\section{After REAC-lithium therapy}

Tables 2-5 and Figures 1-4 compare the overall mood episodes (manic and depressive) per Group before and after REAC-lithium treatment. In Group 1, three manic and one depressive episode occurred. In terms of percentages, there were 0.1 depressive episodes per patient and $0.8 \pm 0.4$ excitative episodes per patient, which was statistically significant in comparison with previous treatments $(P<0.001$ [t-test $])$. Manic episodes occurred during months 19, 22, and 23 of REAC-lithium treatment. All manic episodes were mild (YMRS scores of 24, 23, and 23, respectively), and their mean duration was $8.0 \pm 0.9$ weeks. The lithium dose was increased by approximately $20 \%$, and valproic acid $1000 \mathrm{mg} /$ day and zuclopenthixol $100 \mathrm{mg} /$ day in one case and zuclopenthixol $125 \mathrm{mg} /$ day and clonazepam $6 \mathrm{mg}$ /day in the remaining two patients. The depressive episode, classified mild (Hamilton Depression Rating Scale $=21$ ) occurred during month 23 of treatment with REAC-lithium treatment and was resolved by means of augmentation with bupropion $300 \mathrm{mg} /$ day over a sixweek period. In Group 2, only two manic episodes occurred during months 16 and 19 of REAC-lithium treatment. In terms of percentages, there were $0.11 \pm 0.2$ excitative episodes per patient, which was statistically significant in comparison with previous treatments $(P<0.001[t$-test $])$. Both were mild $($ YMRS $=22)$ with a mean length of $7.2 \pm 0.3$ weeks and were treated with valproate $1000 \mathrm{mg} /$ day and zuclopenthixol (mean dose $87.5 \pm 17.7 \mathrm{mg} /$ day). In Group 3, the one manic episode which occurred during month 17 of REAC-lithium treatment was mild (YMRS $=25$ ), with a length of 8.0 weeks and treatment with zuclopenthixol $75 \mathrm{mg} /$ day and clonazepam $6 \mathrm{mg}$ / day. In terms of percentages, there were 0.4 excitative episodes per patient, which was statistically significant in comparison with previous treatments $(P=0.000[t$-test $])$ In Group 4 , one manic episode occurred during month 13 of REAClithium treatment and was mild (YMRS $=24$ ) with a length of 10.0 weeks and treatment with zuclopenthixol $75 \mathrm{mg} /$ day and valproate $1000 \mathrm{mg} /$ day. In terms of percentages, there

Table 2 Demographic statistical, and mean values for the manic and depressive episodes, before and after REAC-lithium treatment in Group I patients

\begin{tabular}{lll}
\hline Group I $(\mathbf{n}=\mathbf{8})$ & Lithium & REAC-Lithium \\
$\mathbf{7}$ males [BD, I] & & \\
I females [BD, I] & & \\
\hline Excitative episodes & $2.1 \pm 0.6$ & $0.8 \pm 0.4$ \\
Depressive episodes & $3 \pm 0.7$ & $0.1 \pm 0^{*}$ \\
\hline
\end{tabular}

${ }^{*} \mathrm{t}$-Test: $\mathrm{t}=11,718, \mathrm{DF} 14, P=0.000$.

Abbreviations: BD, bipolar disorder; REAC, radioelectric asymmetric brain stimulation device.
Table 3 Demographic statistical, and mean values for the manic and depressive episodes, before and after REAC-lithium treatment in Group 2 patients

\begin{tabular}{lll}
\hline Group $2(\mathbf{n}=$ I4) & Lithium & REAC-Lithium \\
$\mathbf{7}$ males (4 BD I and 3 BD II) & & \\
$\mathbf{7}$ females (all BD II) & & \\
\hline Excitative episodes & $2.4 \pm 0.6$ & $0.1 \pm 0.2$ \\
Depressive episodes & $3.9 \pm 0.7$ & $0 \pm 0^{*}$ \\
\hline
\end{tabular}

${ }^{*} \mathrm{t}$-Test: $\mathrm{t}=20.846, \mathrm{DF} 26, P=0.000$

Abbreviations: $B D$, bipolar disorder; REAC, radioelectric asymmetric brain stimulation device.

were 0.1 excitative episodes per patients, which was statistically significant in comparison with previous treatments $(P=0.000[t$-test $])$.

No hospitalizations occurred during REAC-lithium treatment observation periods. The safety and tolerability profiles of combined REAC-lithium treatment were favorable in all patients. The overall clinical results before and after REAC augmentation of lithium are shown in Tables 2-5 and Figures 1-4. Rates of hospitalization before and after REAC augmentation of lithium are shown in Table 6.

\section{Discussion}

The major finding of this research was the superior effectiveness of REAC-lithium treatment in comparison with the corresponding previous treatment periods, in terms of depressive rather than manic recurrences, in particular with respect to the comparison with lithium alone. It is interesting to note that this seems to be related to the REAC optimization of global brain functioning that is probably disrupted in bipolar depression as a consequence of previous manic-hypomanic episodes. The relevance of these results should be considered in relation to the well known drug resistance of this clinical disorder. The antidepressant activity of REAC-lithium treatment is the consequence of the action of the REAC device which, through the modulation of inter-intra neuronal microcurrents and microelectromagnetic fields, ${ }^{31}$ enables potentiation of the neurobiological activity of lithium.

Table 4 Demographic statistical, and mean values for the manic and depressive episodes, before and after REAC-lithium treatment in Group 3 patients

\begin{tabular}{lll}
\hline Group $3(\mathbf{n}=\mathbf{2 5})$ & Lithium & REAC-Lithium \\
I I males [4 BD I and 7 BD II] & & \\
I 4 females [3 BD I and I I BD II] & & \\
\hline Excitative episodes & $2.6 \pm 0.8$ & $0.4 \pm 0$ \\
Depressive episodes & $3.6 \pm 0.9$ & $0 \pm 0^{*}$ \\
\hline
\end{tabular}

" $t$-Test: $\mathrm{t}=20.000, \mathrm{DF} 48, P=0.000$.

Abbreviations: $\mathrm{BD}$, bipolar disorder; REAC, radioelectric asymmetric brain stimulation device. 
Table 5 Demographic statistical, and mean values for the manic and depressive episodes, before and after REAC-lithium treatment in Group 4 patients

\begin{tabular}{lll}
\hline Group $4(n=9)$ & Lithium & REAC-Lithium \\
$\mathbf{3}$ males [I BD I and 2 BD II] & & \\
$\mathbf{6}$ females [2 BD I and 4 BD II] & & \\
\hline Excitative episodes & $2.6 \pm 1.1$ & $0.1 \pm 0$ \\
Depressive episodes & $3.7 \pm 1$ & $0 \pm 0^{*}$ \\
\hline
\end{tabular}

"t-Test: $t=11.100, D F 16, P=0.000$.

Abbreviations: $B D$, bipolar disorder; REAC, radioelectric asymmetric brain stimulation device.

Other work of ours in progress conducted with functional magnetic resonance images in healthy subjects asked to perform finger tapping has demonstrated a significant reduction of the cerebral areas involved in the planning, control, execution, and stopping of this motor task (Figures 5A and 5B). Thus, according to our studies, REAC allows for better neural synchronization, such that less cerebral activity is needed to do the same movement. This deep cerebral activity of synchronization, as observed in normal subjects, is probably the main mechanism through which the REAC stabilizes the pathologic mood fluctuations in patients with bipolar disorder, so as to prevent morbid episodes, increase the duration of the so-called "free interval", reduce the need for drug adjustments, to minimize the risk of hospitalizations. Our work shifts the focus from the biochemistry to the bioelectricity of the brain; it is important to emphasize that REAC seems synergic with lithium, which is not a drug but an ion, with an microelectric charge and a relative microelectromagnetic field. An interesting feature is that almost all patients included in the follow-up with REAClithium treatment experienced longer free intervals compared with previous treatment periods. REAC-lithium treatment seems to be a good strategy suitable for the treatment of free intervals in bipolar disorder. Until now, the free interval in a bipolar patient is defined as status free from depression and mania, but more recently it has been suggested that the free interval is not free. It is known that, after some time, the majority of bipolar patients show various clinical signs which correspond closely with requests for therapeutic and/or dose adjustment, and this occurs even if depressive or manic

Table 6 Rate of hospitalization before and after REAC augmentation of lithium

\begin{tabular}{lllll}
\hline & Group I & Group 2 & Group 3 & Group 4 \\
\hline Lithium & $7(5 \mathrm{D}, 2 \mathrm{M})$ & $9(9 \mathrm{D})$ & $7(4 \mathrm{D}, 3 \mathrm{M})$ & $2(\mathrm{I}, \mathrm{D}, \mathrm{M})$ \\
REAC- & 0 & 0 & 0 & 0 \\
lithium & & & & \\
\hline
\end{tabular}

Abbreviation: $D$, depressive episodes; M, excitative episodes; REAC, radioelectric asymmetric brain stimulation device.

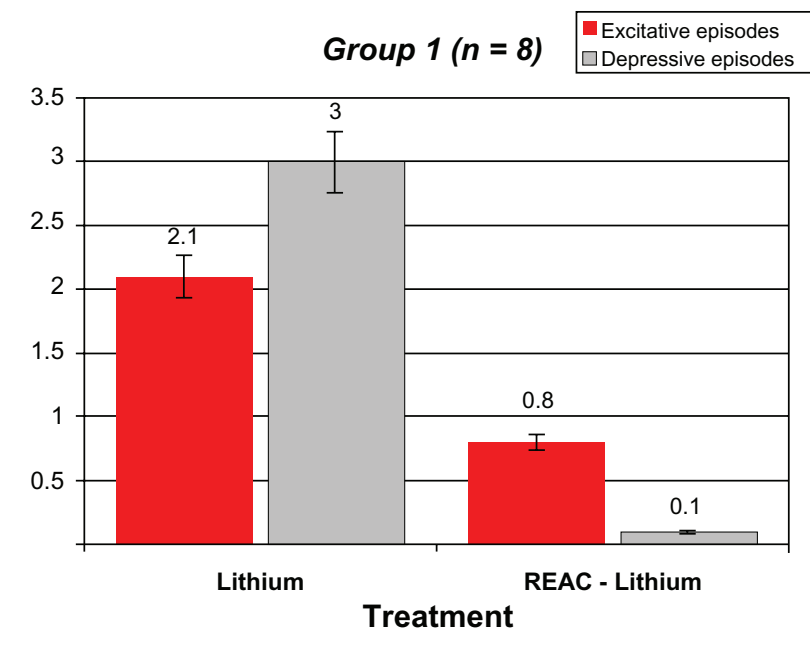

Figure I Demographic statistical, and mean values for the manic and depressive episodes, before and after REAC-lithium treatment in group I patients. Abbreviation: REAC, radioelectric asymmetric brain stimulation device.

mood phases are not ongoing. This phenomenon can be explained only and exclusively with the persistence of a global neurobiological instability, for which the quantitative and qualitative boundaries are unknown. Therefore, improvement of the free interval becomes the main objective in treatment of bipolar disorder. The role of REAC-lithium treatment during intercritic periods can be considered as a therapeutic effect not related to its antidepressant or antimanic activity, but strictly related to the global optimization of brain functioning, broadening its action target so as to spread to temporal prolongation, and to qualitative improvement of the free interval. Consequently, if improvement of the state of the bipolar patient during the intercritic phases is associated with a significantly reduced risk of depressive and manic recurrences, the intercritic period should become the primary

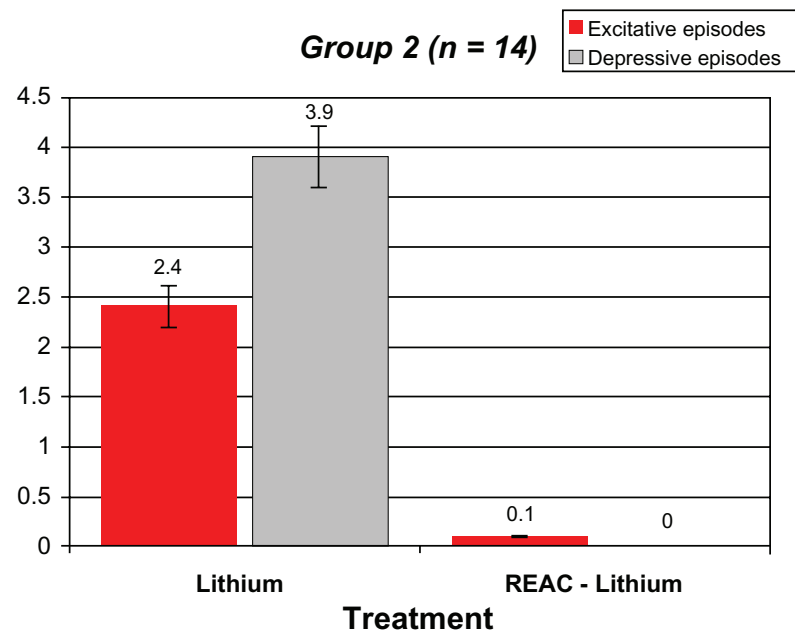

Figure 2 Demographic statistical, and mean values for the manic and depressive episodes, before and after REAC-lithium treatment in group 2 patients. Abbreviation: REAC, radioelectric asymmetric brain stimulation device. 


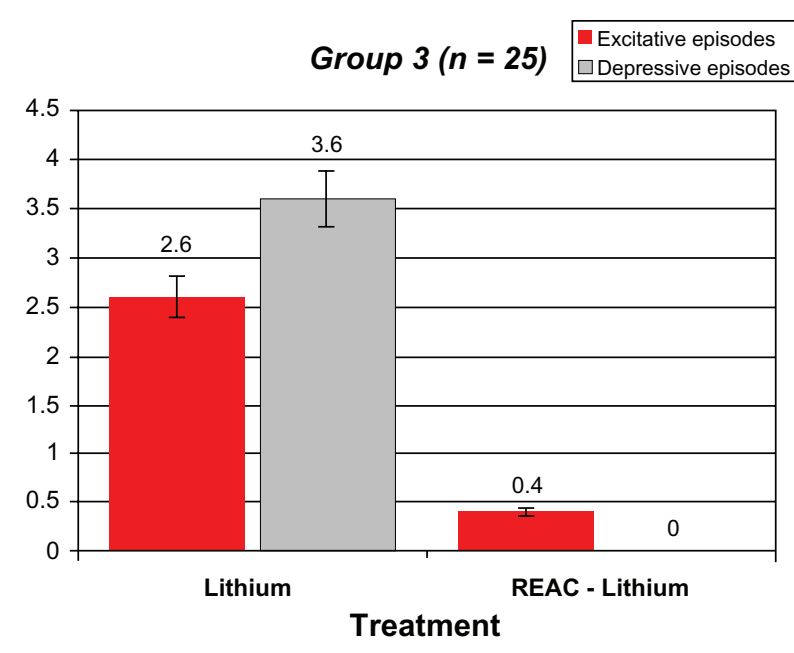

Figure 3 Demographic statistical, and mean values for the manic and depressive episodes, before and after REAC-lithium treatment in group 3 patients.

Abbreviation: REAC, radioelectric asymmetric brain stimulation device.

objective in treatment of bipolar disorder. Furthermore, the preventive action of REAC-lithium, both in terms of manic mood and depressive recurrences, seems to be progressively stronger over time. In fact, all the mood episodes happened within 24 months from the beginning of REAC-lithium treatment. This feature seems to be in conflict with the observations drawn from olanzapine and risperidone (but not for quetiapine), for which the clinical experience shows increasing development of therapeutic tolerance phenomena. In fact, the depressive episodes of bipolar disorder seem to predispose to development of new depressive episodes, and after some time, produce a chronic hypotimic picture. Bipolar depression is a clinical dilemma because affected patients

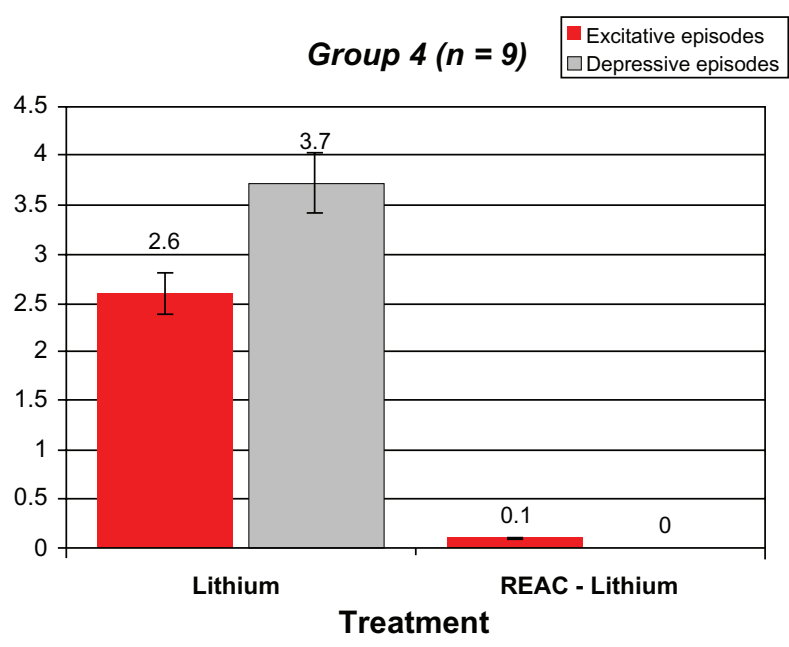

Figure 4 Demographic statistical, and mean values for the manic and depressive episodes, before and after REAC-lithium treatment in group 4 patients.

Abbreviation: REAC, radioelectric asymmetric brain stimulation device.
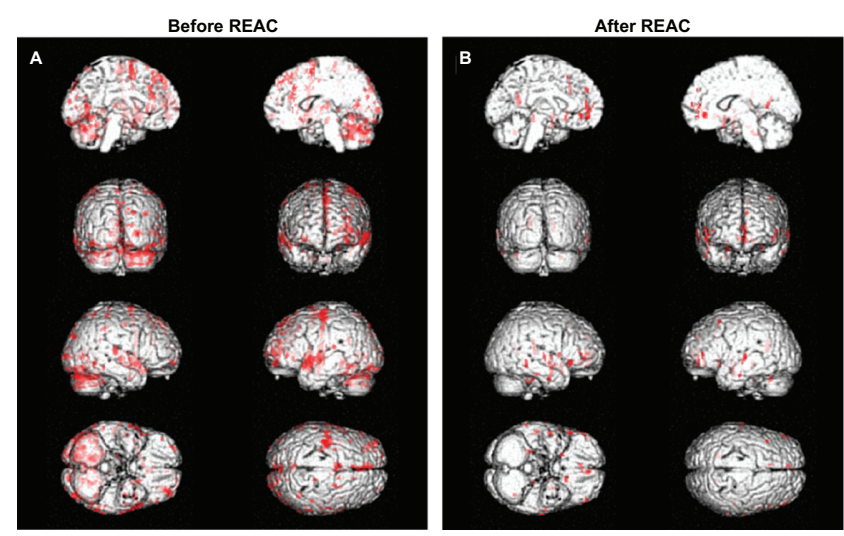

Figure 5 Functional magnetic resonance images in healthy subjects asked to perform finger tapping. (A) Before REAC-NPO treatment and (B) after REAC-NPO treatment.

Abbreviations: NPO, neuropostural optimization; REAC, radioelectric asymmetric brain stimulation device.

are usually poor responders to traditional antidepressant treatments, ie, tricyclic antidepressants, selective serotonin reuptake inhibitors, and selective norepinephrine reuptake inhibitors. Furthermore, with an increased risk of manic switches, cycles speed up, and there is progressive prognostic deterioration. ${ }^{14,32}$ Therefore, in light of these real difficulties of treatment, it is very important to prevent the depressive episodes of bipolar disorder, and use of REAC is a very promising therapeutic modality. Small sample size, a naturalistic and flexible dose design, maintenance of lithium, and lack of accurate assessments were the main limitations of this study. Nevertheless, we suggest that naturalistic studies and not randomized clinical trials supply a correct picture of the routine medical practice with real patients and real-life situations. ${ }^{33}$ Moreover, the long duration of observation can better clarify the clinical evolution of bipolar disorder. The maintenance of lithium treatment associated with REAC is due to ethical and legal considerations in relation to the fact that bipolar disorder is a severe condition (ie, carries a suicide rate $>20 \%$ ), and REAC is effective, albeit not well studied as yet in Italy.

\section{Acknowledgments}

The authors thank Dr Matteo Lotti Margotti for the data analysis, and Lucia Aravagli and Stefania Bini of the RinaldiFontani Institute, Department of Neuro-Psycho-Physio Pathology, Florence, Italy, for their helpful discussions.

\section{Disclosure}

Salvatore Rinaldi and Vania Fontani are the inventors of the radioelectric asymmetric conveyor. 


\section{References}

1. Akiskal HS. The prevalent clinical spectrum of bipolar disorders: Beyond DSM-IV. J Clin Psychopharmacol. 1996;16(2 Suppl 1): 4S-14S.

2. Angst J, Merikangas K. The depressive spectrum: diagnostic classification and course. J Affect Disord. 1997;45(1-2):31-39.

3. Angst J. The bipolar spectrum. Br J Psychiatry. 2007;190:189-191.

4. Goodwin FK, Jameson KR. Manic-Depressive Illness: Bipolar Disorders and Recurrent Depression. New York, NY: Oxford University Press; 1990.

5. Calabrese JR, Shelton MD, Rapport DJ, Kimmel SE. Bipolar disorders and the effectiveness of novel anticonvulsants. J Clin Psychiatry. 2002; 63 Suppl 3:5-9.

6. Berk M, Berk L. Mood stabilizers and treatment adherence in bipolar disorder: addressing adverse events. Ann Clin Psychiatry. 2003; 15(3-4):217-224.

7. Chue P, Kovacs CS. Safety and tolerability of atypical antipsychotics in patients with bipolar disorder: prevalence, monitoring and management. Bipolar Disord. 2003;5 Suppl 2:62-79.

8. Grof P. Selecting effective long-term treatment for bipolar patients: monotherapy and combinations. J Clin Psychiatry. 2003;64 Suppl 5: 53-61.

9. Perugi G, Micheli C, Akiskal HS, et al. Polarity of the first episode, clinical characteristics, and course of manic depressive illness: a systematic retrospective investigation of 320 bipolar I patients. Compr Psychiatry. 2000;41(1):13-18.

10. Judd LL, Akiskal HS. Depressive episodes and symptoms dominate the longitudinal course of bipolar disorder. Curr Psychiatry Rep. 2003; 5(6):417-418.

11. Sienaert P, Vansteelandt K, Demyttenaere K, Peuskens J. Ultra-brief pulse ECT in bipolar and unipolar depressive disorder: differences in speed of response. Bipolar Disord. 2009;11(4):418-424.

12. Grunhaus L, Schreiber S, Dolberg OT, Hirshman S, Dannon PN. Response to ECT in major depression: are there differences between unipolar and bipolar depression? Bipolar Disord. 2002;4 Suppl 1: 91-93.

13. Post RM. The impact of bipolar depression. J Clin Psychiatry. 2005; 66 Suppl 5:5-10.

14. Thase ME. Bipolar depression: issues in diagnosis and treatment. Harv Rev Psychiatry. 2005;13(5):257-271.

15. Bhagwagar Z, Goodwin GM. Lamotrigine in the treatment of bipolar disorder. Expert Opin Pharmacother. 2005;6(8):1401-1408.

16. Montes JM, Saiz-Ruiz J, Lahera G, Asiel A. Lamotrigine for the treatment of bipolar spectrum disorder: a chart review. J Affect Disord. 2005;86(1):69-73.

17. Gao K, Calabrese JR. Newer treatment studies for bipolar depression. Bipolar Disord. 2005;7 Suppl 5:13-23.

18. Calabrese JR, Hirschfeld RM, Frye MA, Reed ML. Impact of depressive symptoms compared with manic symptoms in bipolar disorder: results of a US community-based sample. J Clin Psychiatry. 2004; 65(11):1499-1504.

19. Dean BB, Gerner D, Gerner RH. A systematic review evaluating health-related quality of life, work impairment, and healthcare costs and utilization in bipolar disorder. Curr Med Res Opin. 2004;20(2): $139-154$.
20. Rinaldi S, Fontani V, Moretti E, et al. A new approach on stress-related depression and anxiety: neuro-psycho-physical-optimization with radio electric asymmetric-conveyer. Indian J Med Res. 2010;132:189-194.

21. Rinaldi S, Fontani V, Aravagli L, Mannu P. Psychometric evaluation of a radio electric auricular treatment for stress related disorders: a double-blinded, placebo-controlled controlled pilot study. Health Qual Life Outcomes. 2010;8(1):31.

22. Rinaldi S, Fontani V, Aravagli L, Margotti ML. Psychological and symptomatic stress-related disorders with radio-electric treatment: psychometric evaluation. Stress and Health. January 20, 2010. Available from: http://onlinelibrary.wiley.com/doi/10.1002/smi.1298/abstract. Accessed May 26, 2011.

23. Mannu P, Rinaldi S, Fontani V, Castagna A, Lotti Margotti M. Radio electric treatment vs escitalopram in the treatment of panic disorders associated with major depression: an open-label, naturalistic study. Acupunct Electrother Res. 2009;34:135-149.

24. Collodel G, Moretti E, Fontani V, et al. Effect of emotional stress on sperm quality. Indian J Med Res. 2008;128(3):254-261.

25. Castagna A, Rinaldi S, Fontani V, Aravagli L, Mannu P, Margotti ML. Does osteoarthritis of the knee also have a psychogenic component? Psycho-emotional treatment with a radio-electric device vs intraarticular injection of sodium hyaluronate: an open-label, naturalistic study. Acupunct Electrother Res. 2010;35(1-2):1-16.

26. Rinaldi S, Fontani V. Inventors Rinaldi S, Fontani V, assignees. Radioelectric asymmetric conveyer for therapeutic use. US patent $7,333,8592001$.

27. Rinaldi S, Fontani V. Inventors Rinaldi S, Fontani V, assignees. Radioelectric asymmetric conveyer for therapeutic use. US patent EP1301241 (B1). October 11, 2006.

28. Yeo DT, Wang Z, Loew W, Vogel MW, Hancu I. Local specific absorption rate in high-pass birdcage and transverse electromagnetic body coils for multiple human body models in clinical landmark positions at 3T. J Magn Reson Imaging. 2011;33(5):1209-1217.

29. Higaki N, Shiba K. Analysis of specific absorption rate and current density in biological tissues surrounding energy transmission transformer for an artificial heart: using magnetic resonance imagingbased human body model. Artif Organs. 2010;34(1):E1-E9.

30. Neubauer G, Preiner P, Cecil S, Mitrevski N, Gonter J, Garn H. The relation between the specific absorption rate and electromagnetic field intensity for heterogeneous exposure conditions at mobile communications frequencies. Bioelectromagnetics. 2009;30(8):651-662.

31. Arribas JI, Calhoun VD, Adali T. Automatic Bayesian classification of healthy controls, bipolar disorder, and schizophrenia using intrinsic connectivity maps from FMRI data. IEEE Trans Biomed Eng. 2010; 57(12):2850-2860.

32. Silverstone P. What is the best way to treat bipolar depression? J Psychiatry Neurosci. 2005;30(5):384.

33. Leichsenring F. Randomized controlled versus naturalistic studies: a new research agenda. Bull Menninger Clin. 2004;68(2):137-151.
Neuropsychiatric Disease and Treatment

\section{Publish your work in this journal}

Neuropsychiatric Disease and Treatment is an international, peerreviewed journal of clinical therapeutics and pharmacology focusing on concise rapid reporting of clinical or pre-clinical studies on a range of neuropsychiatric and neurological disorders. This journal is indexed on PubMed Central, the 'PsycINFO' database and CAS, and is the official
Dovepress

journal of The International Neuropsychiatric Association (INA). The manuscript management system is completely online and includes a very quick and fair peer-review system, which is all easy to use. Visit http://www.dovepress.com/testimonials.php to read real quotes from published authors. 